\title{
Coupling-Efficiency Analysis and Control of Dual Antiresonant Reflecting Optical Waveguides
}

\author{
Yeong-Her Chen, Student Member, IEEE, and Yang-Tung Huang, Member, IEEE
}

\begin{abstract}
We have developed the necessary theory to analyze the coupling efficiency of dual ARROW waveguides. Based on the theory, we have found that the coupling efficiency of dual ARROW waveguides can be flexibly controlled from a $100 \%$ value to zero by simply varying the outermost cladding thickness. BPM simulations also give same results and verify the theory. The theory and simulation results can be efficiently applied to designing an ARROW power divider with any specified power ratio.
\end{abstract}

\section{INTRODUCTION}

C OUPLER DEVICES are key integrated optical components in applications relating to modulators, filters, switches, and power dividers [1]. Coupling problems between two parallel waveguides have been investigated and many analyses are based on coupled mode theory with weakly coupled approximations [1]-[7]. For conventional dual waveguides, the coupling strength is decreasing as an exponential function of the waveguide separation. In other words, the coupling efficiency is controlled by waveguide separation. The fact that small separation regions to keep coupled status and wide separation regions to maintain decoupled status between two waveguides is used to demonstrate the coupler devices in real applications. Coupling and decoupling regions are connected by a bend structure. In order to minimize the propagation loss, the bending degree of connecting region is gradually varied, and therefore it needs a long region.

New types of waveguides, antiresonant reflecting optical waveguides (ARROW), have been proposed and demonstrated [8]-[11]. In comparison with conventional structures, ARROW waveguides utilize antiresonant reflection as the guiding mechanism instead of total internal reflection. With this structure, ARROW devices can have a large core size that is compatible with optical fibers. A directional coupler consisting of two identical ARROW's was recently proposed [10]. The main advantage of an ARROW-based coupler over a conventional waveguide coupler is that its coupling-length is not increasing, but varies as a periodic function with increasing waveguide separation. A similar dual-ARROW stacked structure was demonstrated in 1988 [9], and experimental results showed the decoupled phenomena between two ARROW waveguides. Mann et al. [10] showed that two identical

Manuscript received June 1, 1995; revised February 12, 1996. This work was supported by the National Science Council of the Republic of China under Contract NSC84-8421-E-009-009.

The authors are with the Department of Electronics Engineering and Institute of Electronics, National Chiao Tung University, Hsinchu, Taiwan, R.O.C

Publisher Item Identifier S 0733-8724(96)04604-X.
ARROW structures had a very high coupling efficiency, but Baba et al, showed that similar ARIROW structures had a lower coupling efficiency. The reason and analysis of their difference have not been clearly discussed. In this paper, we develop the necessary theory to describe the coupling efficiency between dual ARROW waveguides to clearly explain the different phenomena of Mann's and Baba's cases.

In Section II, the basic structure is constructed by transverse resonance formulas for guiding layers and antiresonance formulas for the cladding layers. In addition, modal analysis of a dual ARROW coupler is briefly discussed. Dual ARROW waveguides with leaky modes belong to strongly coupled structures. Many coupling analytical methods based on weakly coupled approximation are difficult to apply to strongly coupled waveguides. In Section III, we derive the necessary expressions to describe the coupling efficiency between dual ARROW waveguides for strongly coupled structures. In Section IV, dual ARROW waveguides based on $\mathrm{TiO}_{2} / \mathrm{SiO}_{2} / \mathrm{Si}$ and InGaAsP/InP material systems are analyzed. It can be seen that the coupling efficiencies of dual ARROW waveguides strongly depend on upper cladding thicknesses, and the efficiencies can be controlled from a $100 \%$ value to zero by simply varying the outermost cladding thickness. The analytical results clearly explain the different phenomena of Mann's and Baba's cases described above. We also use BPM simulations to obtain the same results and verify our theory. The theory and simulation results can be efficiently applied to designing an ARROW power divider with any specified power ratio.

\section{Modal ANalysis of Dual ARrow Devices}

The configuration of dual-ARROW coupling basic structure $\left(n_{a} / n_{l 1} / n_{h 1} / n_{g 1} / n_{h 1} / n_{\text {sep }} / n_{h 2} / n_{g 2} / n_{h 2} / n_{l 2} / n_{s}\right)$ is depicted in Fig. 1(a), which consists of two ARROW waveguides and a separation cladding layer. The core layers of two ARROW waveguides are with low refractive indexes of $n_{g 1}$ and $n_{g 2}$, and thicknesses of $d_{g 1}$ and $d_{g 2}$, respectively. The upper waveguide is sandwiched between two high index $\left(n_{h 1}\right)$ cladding layers of thickness $d_{h 1}^{\prime}$ and $d_{h 1}$, and a low index $\left(n_{l 1}\right)$ upper cladding layer of thickness $d_{l 1}$ is grown atop them. The lower waveguide is sandwiched between two high index $\left(n_{h 2}\right)$ cladding layers of thickness $d_{h 2}$ with a low index $\left(n_{l 2}\right)$ lower cladding layer of thickness $d_{l 2}$. Between two ARROW waveguides there is a separation cladding layer

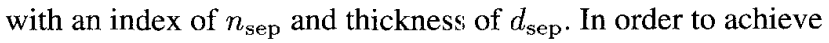
low loss, the core layer of the lower ARROW waveguide must satisfy the transverse resonance condition. The thickness $d_{g 2}$ 


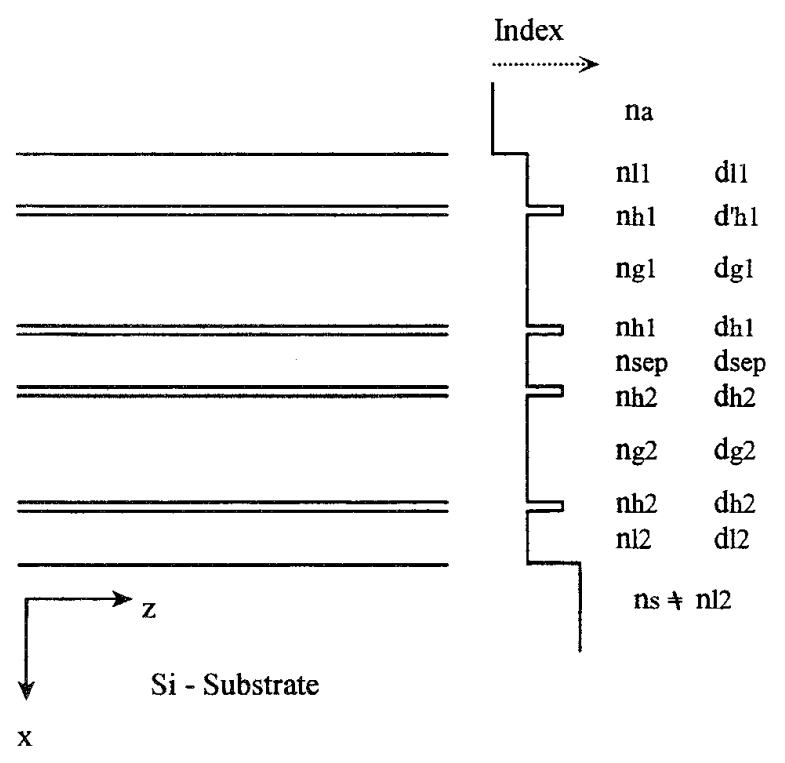

(a)

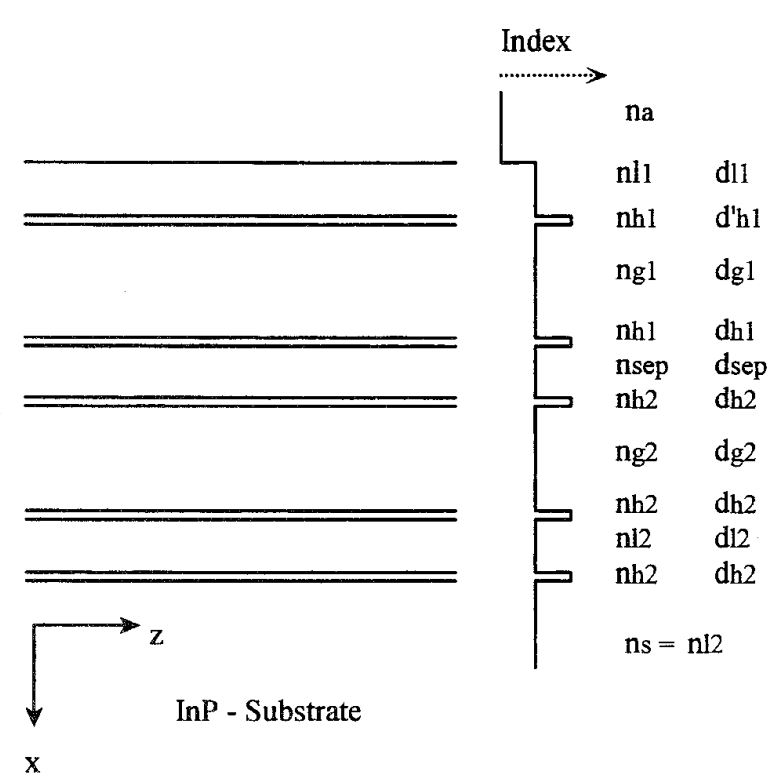

(b)

Fig. 1. (a) Basic coupling structure of the proposed based on dual-ARROW waveguides with $\mathrm{Si}$ substrate, (b) Basic coupling structure of the proposed based on dual-ARROW waveguides with InP substrate.

is given as [8]

$$
d_{g 2}=\frac{\lambda}{2 n_{g 2}}\left[1-\left(\frac{n_{g 1}}{n_{g 2}}\right)^{2}+\left(\frac{\lambda}{2 n_{g 2} d_{g 1}}\right)^{2}\right]^{-1 / 2}
$$

Except for the upper cladding layers of $d_{l 1}$ and $d_{h 1}^{\prime}$, all cladding layers satisfy the antiresonance condition as

$$
d_{j}=\frac{\lambda}{4 n_{j}}\left[1-\left(\frac{n_{g 1}}{n_{j}}\right)^{2}+\left(\frac{\lambda}{2 n_{j} d_{g 1}}\right)^{2}\right]_{P_{j}}^{-1 / 2}\left(2 P_{j}+1\right),
$$

where $j=h 1$ (and $h 2$ ), sep, and $l 1$ (and $l 2$ ), representing corresponding high-index, separation, and low-index cladding layers, respectively. Since the coupling strength between dual ARROW waveguides strongly depends on the degree of their symmetry, we can easily adjust the thickness of the upper cladding layer to change the degree of their symmetry and thus vary the coupling efficiency.

In our analysis, we restrict ourselves to the case of TEpolarized field. By the characteristic matrix method, the dispersion relation in multilayer slab is given as [10]

$$
k_{a, x}\left(m_{11}+k_{s, x} m_{12}\right)+\left(m_{21}+k_{s, x} m_{22}\right)=0
$$

where $k_{a, x}$ and $k_{s, x}$ represent the transverse propagation constant in air and the substrate, given as

$$
\begin{aligned}
& k_{a, x}=\sqrt{k_{0}^{2} n_{a}^{2}-\beta^{2}}=k_{0} \sqrt{n_{a}^{2}-N_{\mathrm{eff}}^{2}} \\
& k_{s, x}=\sqrt{k_{0}^{2} n_{s}^{2}-\beta^{2}}=k_{0} \sqrt{n_{s}^{2}-N_{\mathrm{eff}}^{2}}
\end{aligned}
$$

$k_{0}$ is the wavenumber in air, $n_{a}$ is the index of air, and $N_{\text {eff }}$ is the effective index of the propagation wave. The characteristic matrix $\mathbf{M}$ with elements $m_{i j}$ is given as

$$
\begin{aligned}
\mathbf{M} & =\left[\begin{array}{ll}
m_{11} & m_{12} \\
m_{21} & m_{22}
\end{array}\right]=\prod_{i} \mathbf{M}_{i} \\
& =\prod_{i}\left[\begin{array}{cc}
\cos k_{i, x} d_{i} & \left(j / k_{i, x}\right) \sin k_{i, x} d_{i} \\
j k_{i, x} \sin k_{i, x} d_{i} & \cos k_{i, x} d_{i}
\end{array}\right] .
\end{aligned}
$$

The $i$ indicates each layer except for air and substrate. When $n_{l 2}=n_{s}$, such as in InGaAsP material system, a destructive interference layer with index of $n_{h 2}$ and thickness of $d_{h 2}$ between this low index cladding layer $\left(n_{l 2}\right)$ and substrate $\left(n_{s}\right)$ should be added to maintain low radiation loss into the substrate. This structure $\left(n_{a} / n_{l 1} / n_{h 1} / n_{g 1} / n_{h 1} / n_{\text {sep }} / n_{h 2} / n_{g 2} / n_{h 2} / n_{l 2} / n_{h 2} / n_{s}\right) \quad$ is shown in Fig. 1(b).

\section{COUPLING-EFFICIENCY ANALYSIS}

Coupling between two parallel waveguides has been widely investigated [1]-[7]. Most analyses still employ the coupled mode formulations developed by Marcuse, Taylor, and Yariv [3] [7]. Their results are accurate only in weakly coupling situations. For dual-ARROW structures with leaky modes, the strong coupling between two waveguides must be considered. We employ the method based on interference of the even and odd lowest order modes to analyze the coupling efficiency of a dual-ARROW structure [12]. Although there might exist some other higher order modes with low propagation loss, only these two lowest order modes can be efficiently excited by the fundamental mode from a single-mode fiber (or waveguide) input-coupling, and this will be discussed in detail in another paper. The field of a dual-ARROW guided system can be expressed as

$$
\begin{aligned}
E(x, z)= & A_{e} E_{e}(x) \exp \left(-j k_{0} N_{e} z\right) \\
& +A_{o} E_{o}(x) \exp \left(-j k_{0} N_{o} z\right) \\
H(x, z)= & A_{e} H_{e}(x) \exp \left(-j k_{0} N_{e} z\right) \\
& +A_{o} H_{o}(x) \exp \left(-j k_{0} N_{o} z\right)
\end{aligned}
$$




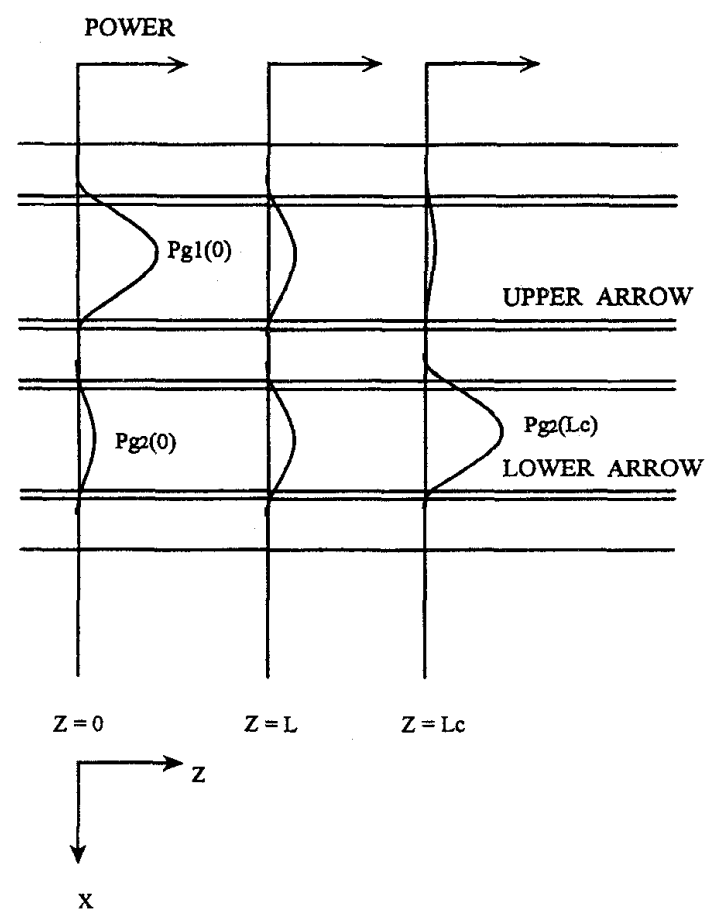

Fig. 2. Schematic diagram for optical power coupling from the upper waveguide to the lower waveguide.

where $A_{e}$ and $A_{o}$ are amplitudes of the normalized even and odd modes with effective indexes $N_{e}$ and $N_{o}$, respectively. $E_{e},\left(H_{e}\right)$ and $E_{o},\left(H_{o}\right)$ are the normalized electric (magnetic) fields of even and odd modes, respectively. In TE modes, the power density propagating along the waveguide is obtained as

$$
\begin{aligned}
S_{z}(x, z)= & \frac{1}{2} \operatorname{Re}\left[\left(\vec{E} \times \vec{H}^{*}\right) \cdot \hat{a}_{z}\right] \\
= & \frac{1}{2} \operatorname{Re}\left[E_{y}(x, z) H_{x}^{*}(x, z)\right] \\
=\frac{1}{2}\{ & A_{e}^{2} E_{e}(x) H_{e}^{*}(x)+A_{o}^{2} E_{o}(x) H_{o}^{*}(x) \\
& \quad+A_{e} A_{o} \cdot\left[E_{e}(x) H_{o}^{*}(x)+E_{o}(x) H_{e}^{*}(x)\right] \\
& \left.\cdot \cos \left[k_{0}\left(N_{e}-N_{o}\right) z\right]\right\} .
\end{aligned}
$$

The even and odd modes are orthonormal with respect to the Poynting power. The wave is normalized as

$$
\begin{aligned}
P & =\int_{-\infty}^{\infty} S_{z}(x, z) d x=P_{g 1}+P_{g 2}+P_{\text {else }} \\
& =A_{e}^{2}+A_{o}^{2}=1,
\end{aligned}
$$

where

and

$$
P_{g 1}(z)=\int_{g 1} S_{z}(x, z) d x, P_{g 2}(z)=\int_{g 2} S_{z}(x, z) d x
$$

$$
P_{\text {else }}(z)=\int_{\text {else }} S_{z}(x, z) d x
$$

$P_{g 1}$ is the guided power in the upper waveguide $g 1, P_{g 2}$ is the guided power in the lower waveguide $g 2$, and $P_{\text {else }}$ represents the remaining power in the region outside guides $g_{1}$ and $g_{2}$.
Light is launched to the upper ARROW waveguide in Fig. 2 at the reference plane $z=0$ such that there is a reference power $P_{g 1}$ guided in the upper waveguide. To calculate the coupling efficiency $C(z)$ from the upper to the lower waveguides at any $z$ position, we must investigate the power variation $\Delta P_{g 2}$ in the lower waveguides. By combining (10) and (11), the power in the upper ARROW waveguide at a position $z$ is given as

$$
\begin{aligned}
P_{g 1}(z)=P- & {\left[P_{g 2}(z)+P_{\text {else }}(z)\right] } \\
=1- & \left\{A_{e}^{2}\left(1-\xi_{e e}\right)+A_{o}^{2}\left(1-\xi_{o o}\right)\right. \\
& \left.-A_{e} A_{o}\left(\xi_{e o}+\xi_{o e}\right) \cos \left[k_{0}\left(N_{e}-N_{o}\right) z\right]\right\}
\end{aligned}
$$

and the initial guiding power in the upper waveguide at $z=0$ is

$$
\begin{aligned}
P_{g 1}(0)=P- & \left(P_{g 2}(0)+P_{\text {else }}(0)\right) \\
=1- & {\left[A_{e}^{2}\left(1-\xi_{e e}\right)+A_{o}^{2}\left(1-\xi_{o o}\right)\right.} \\
& \left.-A_{e} A_{o}\left(\xi_{\varrho o}+\xi_{o e}\right)\right]
\end{aligned}
$$

where $\xi_{e e}, \xi_{o o}, \xi_{e o}$ and $\xi_{o e}$ are defined as

$$
\begin{aligned}
\xi_{i j} & =\frac{1}{2} \int_{g 1} E_{j}(x) H_{i}^{*}(x) d x \\
& =\left\{\begin{array}{cl}
1-\frac{1}{2} \int_{g 2} E_{j}(x) H_{i}^{*}(x) d x \\
-\frac{1}{2} \int_{\text {else }}^{2} E_{j}(x) H_{i}^{*}(x) d x, & (i=j) \\
-\frac{1}{2} \int_{g 2} E_{j}(x) H_{i}^{*}(x) d x & \\
-\frac{1}{2} \int_{\text {else }} E_{j}(x) H_{i}^{*}(x) d x, & (i \neq j) .
\end{array}\right.
\end{aligned}
$$

To minimize the uncoupled power $P_{g 1}(0)+P_{\text {else }}(0)$, the cross product $A_{e} A_{o}$ is obtained as [12]

$$
A_{e} A_{o}=\frac{\left(\xi_{e o}+\xi_{o e}\right)}{2\left[\left(\xi_{e e}-\xi_{o o}\right)^{2}+\left(\xi_{e o}+\xi_{o e}\right)^{2}\right]^{\frac{1}{2}}}
$$

and the uncoupled is given as

$$
\begin{aligned}
& P_{g 2}(0)+P_{\text {else }}(0) \\
& \quad=1-\frac{1}{2}\left\{\xi_{e e}+\xi_{o o}+\left[\left(\xi_{e e}--\xi_{o o}\right)^{2}+\left(\xi_{e o}+\xi_{o e}\right)^{2}\right]^{\frac{1}{2}}\right\}
\end{aligned}
$$

Since the loss is small in our structure [9], [10], we have

$$
\begin{aligned}
\Delta P_{\text {else }}(z) & =P_{\text {else }}(z)-P_{\text {else }}(0) \ll \Delta P_{g 2}(z) \\
& =P_{g 2}(z)-P_{g 2}(0)
\end{aligned}
$$

and thus

$$
\begin{aligned}
\Delta P_{g 2}(z) & \approx-\Delta P_{g 1}(z)=P_{g 1}(0)-P_{g 1}(z) \\
& =2 A_{e} A_{o}\left(\xi_{e o}+\xi_{o e}\right) \sin ^{2}\left[\frac{k_{0}\left(N_{e}-N_{o}\right)}{2} z\right] .
\end{aligned}
$$

Based on (12)-(18) we obtain the coupling efficiency as

$$
C(z)=\frac{\Delta P_{g 2}(z)}{P_{g 1}(0)}=C_{o} \sin ^{2}\left(\beta_{c} z\right),
$$

where (20) and (21) are shown at the top of the next page and $C_{o}$ is the maximum power coupling efficiency at the couplinglength $z=L_{c}$. In the case that light is launched to the lower ARROW waveguide, the above formulas will also be satisfied if $P_{g 1}$ and $P_{g 2}$ are exchanged with each other. 


$$
\begin{aligned}
C_{o} & =\frac{2\left(\xi_{e o}+\xi_{o e}\right)^{2}}{\left(\xi_{e e}+\xi_{o o}\right)\left[\left(\xi_{e e}-\xi_{o o}\right)^{2}+\left(\xi_{e o}+\xi_{o e}\right)^{2}\right]^{\frac{1}{2}}+\left(\xi_{e e}-\xi_{o o}\right)^{2}+\left(\xi_{e o}+\xi_{o e}\right)^{2}} \\
\beta_{c} & =\frac{k_{0}\left(N_{e}-N_{o}\right)}{2},
\end{aligned}
$$

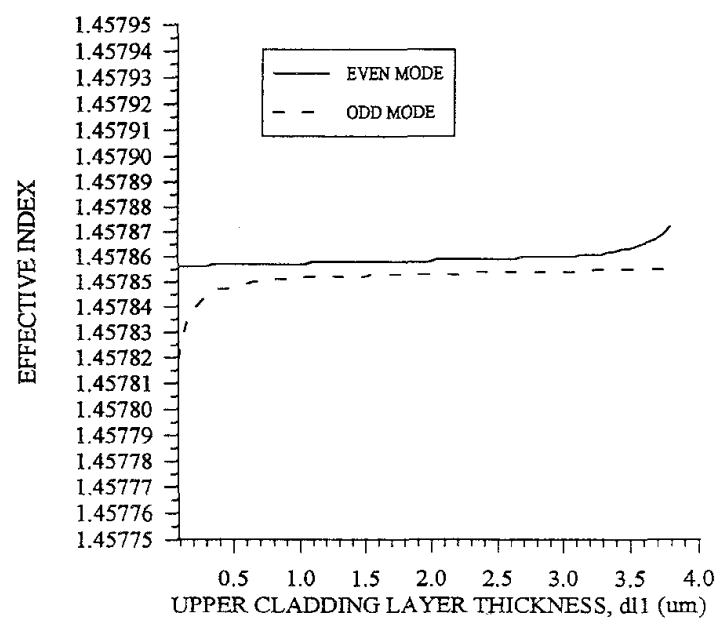

Fig. 3. The dispersion curve for the even and odd mode with corresponding structure in Fig. 1(a).

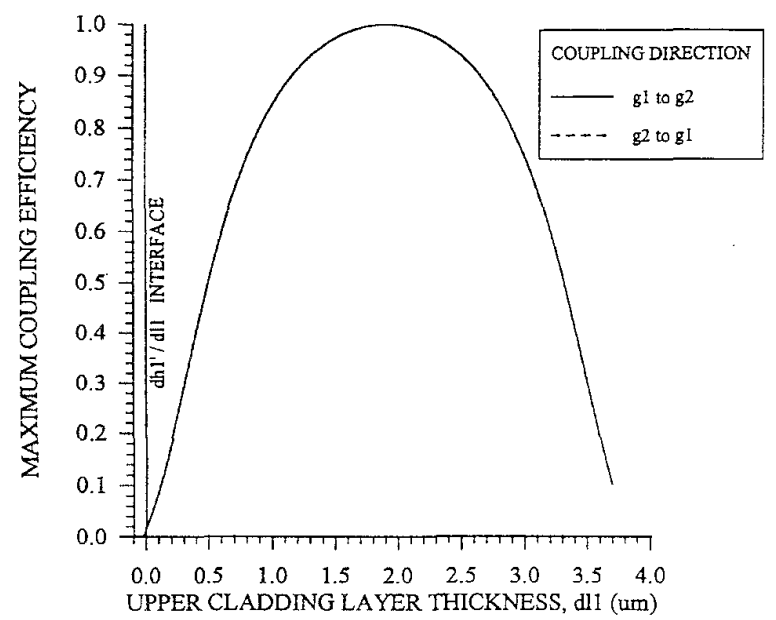

Fig. 4. Maximum coupling efficiency $C_{o}$ versus different upper cladding layer thickness $d_{l 1}$ in Fig. 1(a).

\section{COUPLING-EFFICIENCY CONTROL BY VARYING THE THICKNESSES OF UPPER CLADDING LAYERS}

In this section, we investigate the influence of the upper cladding thickness on the coupling efficiency of dual ARROW waveguides based on the theory developed in Sections II and III, and also use beam propagation method (BPM) to verify the results. The configuration of basic structures based on $\mathrm{Si}$ and InP substrates are depicted in Fig. 1(a) and (b), respectively. For $\mathrm{Si}$ substrate $\left(n_{s}=3.5\right), \mathrm{SiO}_{2}$ with refractive index
1.46 acts as the guide layers, separation layer and low-index cladding layers, i.e., $n_{g 1}=n_{g 2}=n_{\text {sep }}=n_{l 1}=n_{l 2}=1.46$. $\mathrm{TiO}_{2}$ acts as the high-index cladding layers and the refractive index is $n_{h 1}=n_{h 2}=2.3$. The upper waveguide thickness $d_{g 1}$ is $4 \mu \mathrm{m}$ such that this large core is compatible with optical fiber efficiently [8], [9]. Then we have $d_{g 2}=4 \mu \mathrm{m}$, $d_{l 2}=d_{\text {sep }}=2 \mu \mathrm{m}$, and $d_{h 1}=d_{h 2}=0.089 \mu \mathrm{m}$ at the operating wavelength of $\lambda=0.6328 \mu \mathrm{m}$. The thickness variation of the upper cladding layers $d_{l 1}$ and $d_{h 1}^{\prime}$ changes the symmetry degree of dual ARROW waveguides. Symmetric degree will influence the coupling ability in the coupling structures. The dispersion results are illustrated in Fig. 3. In the symmetric region, i.e., $d_{l 1}=d_{l 2}$ and $d_{h 1}^{\prime}=d_{h 1}=d_{h 2}$, both even and odd modes are described by nondispersive (flat) cures which imply symmetric field distributions for two modes, and optical power can efficiently exchange between two ARROW waveguides. In the asymmetric region, i.e., $d_{l 1}$ is highly different from $d_{l 2}$, which implies asymmetric field distributions for two modes, and the coupling ability is very low. Thus based on (20), the maximum coupling efficiency strongly depends on the thickness of the upper cladding layer; the calculated results are shown in Fig. 4. From the calculated results in Fig. 4 , it can be seen that an efficient coupling occurs in the highly symmetric structure of $d_{l 1}=d_{l 2}=2 \mu \mathrm{m}, d_{h 1}^{\prime}=d_{h 1}=d_{h 2}=0.089 \mu \mathrm{m}$ and the maximum coupling efficiency $C_{o}$ is up to $99.87 \%$. The electric field distributions of local normal even and odd modes in this coupling region are shown in Fig. 5(a) as expected. The coupling-length for a maximum coupling efficiency is calculated as $L_{c}=\lambda /\left(2\left|N_{e}-N_{o}\right|\right)=59 \mathrm{~mm}$ from Fig. 3 . In contrast to the efficient coupling region, the decoupled phenomenon occurs at the extreme value of $d_{l 1}=0 \mu \mathrm{m}$ and $d_{h 1}^{\prime}=d_{h 1}-0.059 \mu \mathrm{m}=0.03 \mu \mathrm{m}$. Fig. 5(b) for the field distributions in this decoupling region shows a negligible coupling and the maximum coupling efficiency is obtained as near zero. By means of the thickness adjustment of the upper cladding layer, optical power coupling between two ARROW waveguides can be efficiently controlled. Our analytical results clearly explain the different coupling phenomena of Mann's and Baba's cases [10], [9].

In order to further verify the concept and analysis theory discussed in Sections II and III, we also used BPM to simulate a special structure with informations obtained above. Except for the upper cladding layer thickness, we keep the same parameter values for $\mathrm{SiO}_{2} / \mathrm{TiO}_{2} / \mathrm{Si}$ structure, $n_{l 1}=n_{g 1}=$ $n_{g 2}=n_{l 2}=n_{\text {sep }}=1.46, n_{h 1}=n_{h 2}=2.3, d_{g 1}=$ $d_{g 2}=4 \mu \mathrm{m}, d_{h 1}=d_{h 2}=0.089 \mu \mathrm{m}, d_{\text {sep }}=d_{l 2}=2 \mu \mathrm{m}$, $\lambda=0.6328 \mu \mathrm{m}$. In our BPM simulation structure, we have 


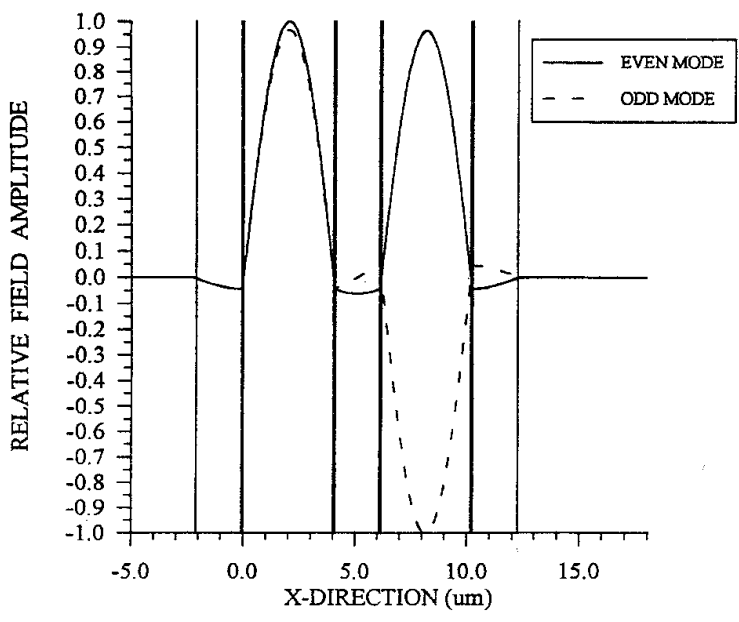

(a)

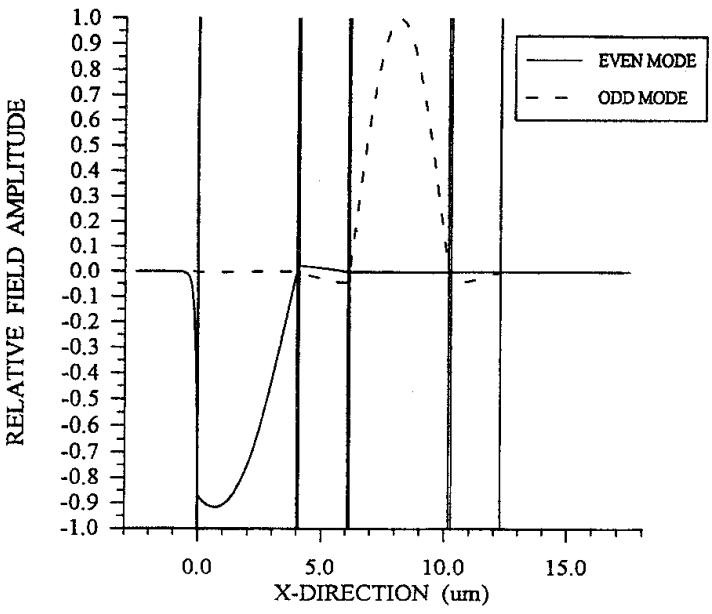

(b)

Fig. 5. $E$-field for $N_{e}$ and $N_{o}$ with $\mathrm{Si}$ substrate: (a) coupling region as in Fig. 1(a): $d_{l 1}=d_{l 2}=2 \mu \mathrm{m}, d_{g 1}=d_{g 2}=4$ $\mu \mathrm{m}, d_{h 1}^{\prime}=d_{h 1}=d_{h 2}=0.089 \mu \mathrm{m}, n_{l 1}=n_{l 2}=n_{g 1}=n_{g 2}=1.46$, $n_{h_{1}} \stackrel{n_{h 2}}{=}=2.3$, and $\lambda=0.6328 \mu \mathrm{m}$, (b) Decoupling region as in Fig. 1(a): $d_{l 1}=0 \mu \mathrm{m}, d_{h_{1}}^{\prime}=0.030 \mu \mathrm{m}, d_{l 2}=2 \mu \mathrm{m}, d_{g 1}=d_{g 2}=4 \mu \mathrm{m}$, $d_{h 1}=d_{h 2}=0.089 \mu \mathrm{m}, n_{l 1}=n_{l 2}=n_{g 1}=n_{g 2}=1.46$, $n_{h 1}=n_{h 2}=2.3$, and $\lambda=0.6328 \mu \mathrm{m}$.

a coupling region with a coupling-length, and then a long decoupling region as shown in Fig. 6(a). From the results shown in Fig. 4, in the coupling region, the thickness of upper cladding layers are $d_{l 1}=d_{l 2}=2 \mu \mathrm{m}$ and $d_{h 1}^{\prime}=d_{h 1}=$ $d_{h 2}=0.089 \mu \mathrm{m}$ to maintain the symmetry such that the energy can efficiently transfer from the upper waveguide to the lower waveguide. After complete energy exchange passing a coupling-length $L_{c}=59 \mathrm{~mm}$, the upper cladding layers are removed to have $d_{l 1}=0 \mu \mathrm{m}$ and $d_{h 1}^{\prime}=0.030 \mu \mathrm{m}$, such that symmetry is completely destroyed and the maximum coupling efficiency is drastically reduced to about zero. In this decoupling region, optical energy can be well confined in the same lower waveguide. The BPM simulation result is illustrated in Fig. 6(b). The result shows that the couplinglength $L_{c}=59 \mathrm{~mm}$ in the coupling region, and after a long distance propagation $\left(>5 L_{c}=295 \mathrm{~mm}\right)$ in the decoupling

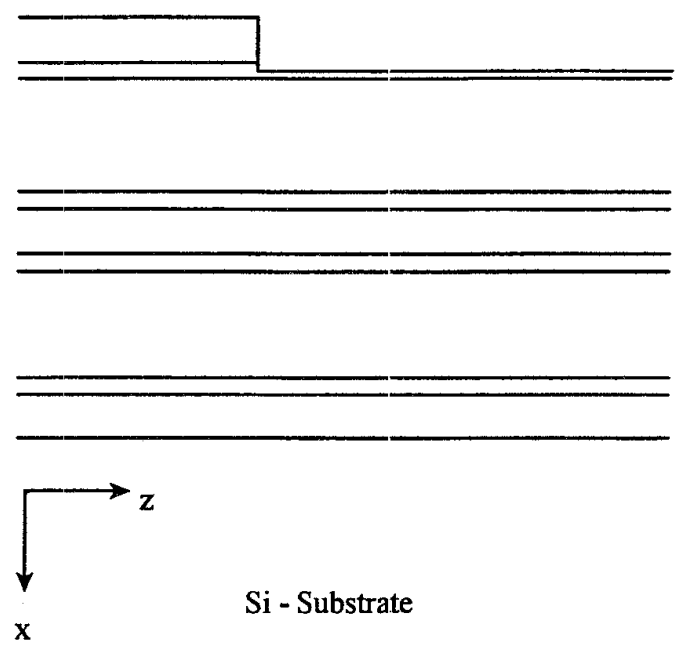

(a)

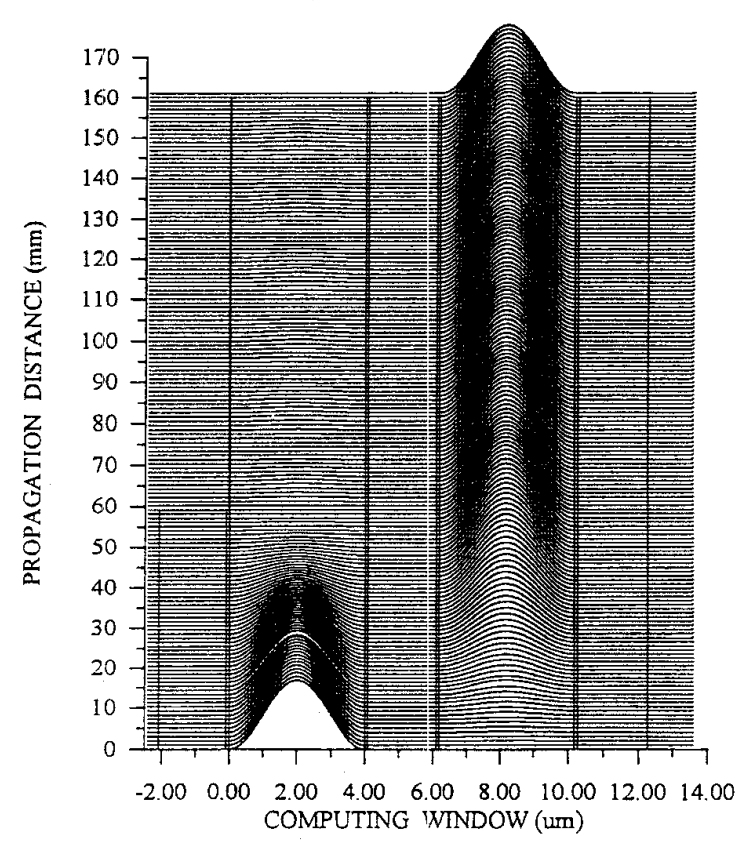

(b)

Fig. 6. (a) The configuration of dual-ARROW switching structure consisting of a coupling and a decoupling region with corresponding parameters in Fig. 5(a) and (b). (b) BPM simulation results with the structure in Fig. 6(a).

region, power is still well confined in the lower ARROW waveguide. It verifies our coupling efficiency analysis.

In InP ( $\left.n_{s}=3.16\right)$ substrate, InP acts as the film material for two guiding layers, the separation layer, and low-index cladding layers, i.e., $n_{g 1}=n_{g 2}=n_{\text {sep }}=n_{l 1}=n_{l 2}=3.16$. $\mathrm{In}_{0.577} \mathrm{Ga}_{0.423} \mathrm{As}_{0.90} \mathrm{P}_{0.10}$ is for high index cladding layers with $n_{h 1}=n_{h 2}=3.55$ at $\lambda=1.55 \mu \mathrm{m}$ [13]. Just like the same reason as in Si substrate case, we design $d_{g 1}=4 \mu \mathrm{m}$ such that this large core is compatible with optical fiber efficiently [11], then $d_{g 2}=4 \mu \mathrm{m}, d_{h 1}=d_{h 2}=0.237 \mu \mathrm{m}$ and $d_{l 2}=2 \mu \mathrm{m}$ can be obtained. We observe the influence of the upper cladding layer thickness in the basic structure as show in Fig. 1(b). The dispersion results are shown in Fig. 7. Fig. 8 represents 


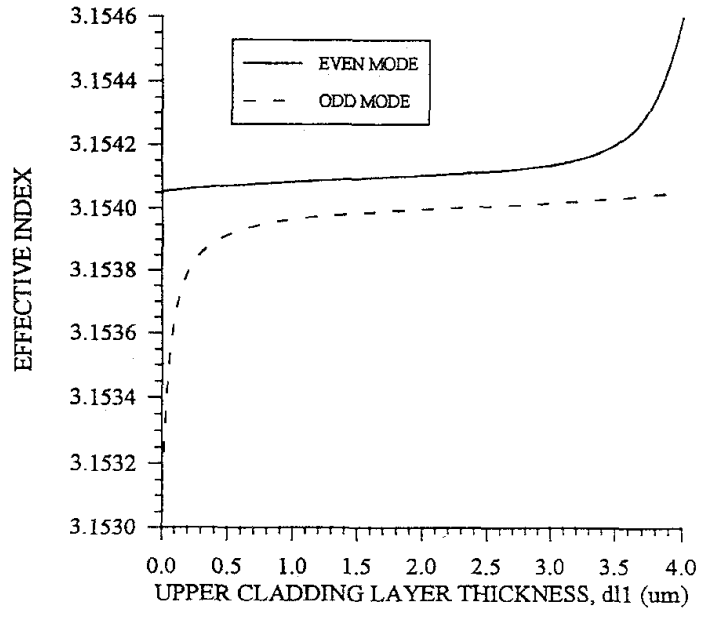

Fig. 7. The dispersion curve for the even and odd mode with corresponding structure in Fig. 1(b).

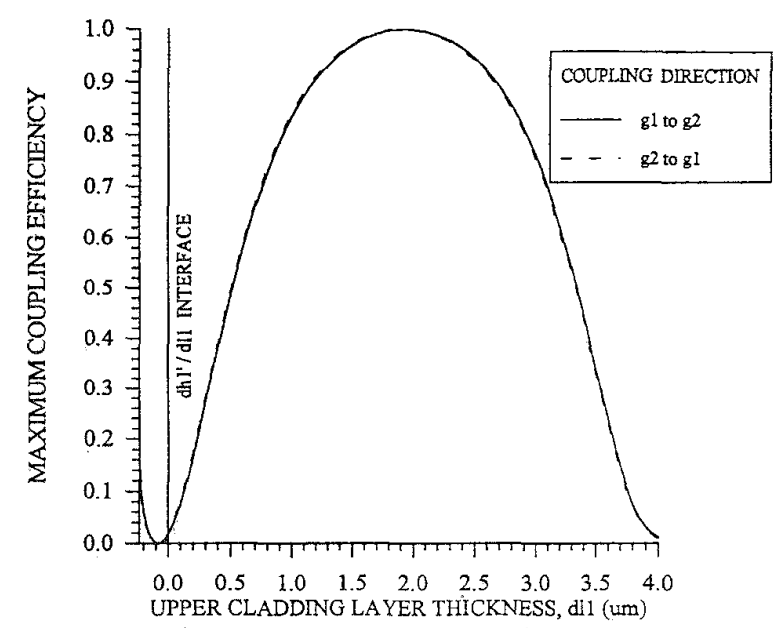

Fig. 8. Power coupling efficiency versus different upper cladding layer thickness $d_{l 1}$ in Fig. 1(b).

the relation between the maximum coupling efficiency and the thickness variation of the upper cladding layers. Similar to Si substrate material, efficient coupling also occurs in the symmetric condition of $d_{l 1}=d_{l 2}=2.0 \mu \mathrm{m}, d_{h 1}^{\prime}=d_{h 1}=$ $d_{h 2}=0.237 \mu \mathrm{m}$ and the maximum coupling efficiency is $C_{o}=99.96 \%$. The field distributions of local normal modes in this case are shown in Fig. 9(a). The coupling-length is obtained as $L_{c}=\lambda /\left(2\left|N_{e}-N_{o}\right|\right)=7.34 \mathrm{~mm}$ from Fig. 7 . Another extreme value for maximum coupling efficiency near zero occurs, when the upper cladding layers are removed such that $d_{l 1}=0 \mu \mathrm{m}$ and $d_{h 1}^{\prime}=d_{h 1}-0.075 \mu \mathrm{m}=0.162 \mu \mathrm{m}$. In this decoupling region, the field profiles of the even and odd modes plotted in Fig. 9(b) again shows a negligible coupling and the maximum coupling efficiency is obtained as near zero. Similar to the devices on Si substrate, we also used the BPM to simulate the wave propagation in a specially designed structure. The BPM simulation structure is depicted in Fig. 10(a), which also consists of a coupling region with $d_{l 1}=d_{l 2}=2.0 \mu \mathrm{m}, d_{h 1}^{\prime}=d_{h 1}=d_{h 2}=0.237 \mu \mathrm{m}$ with a

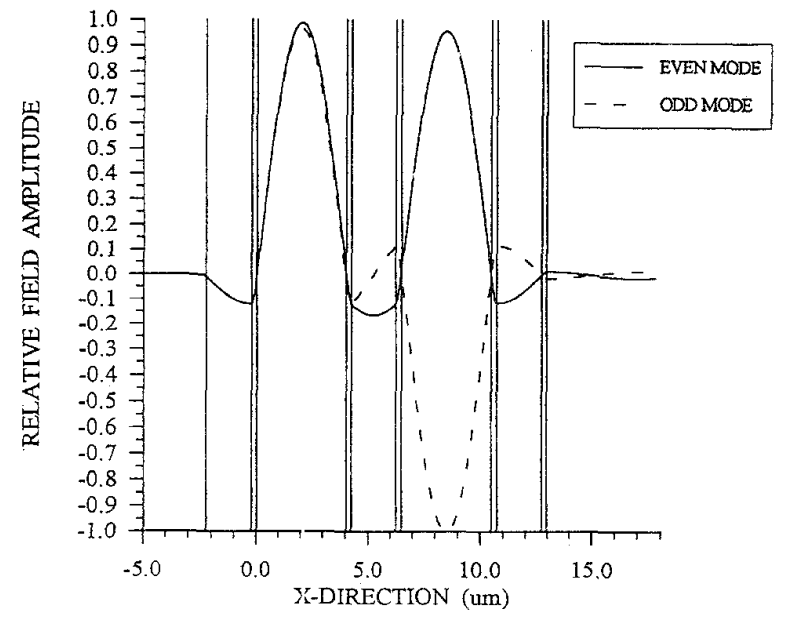

(a)

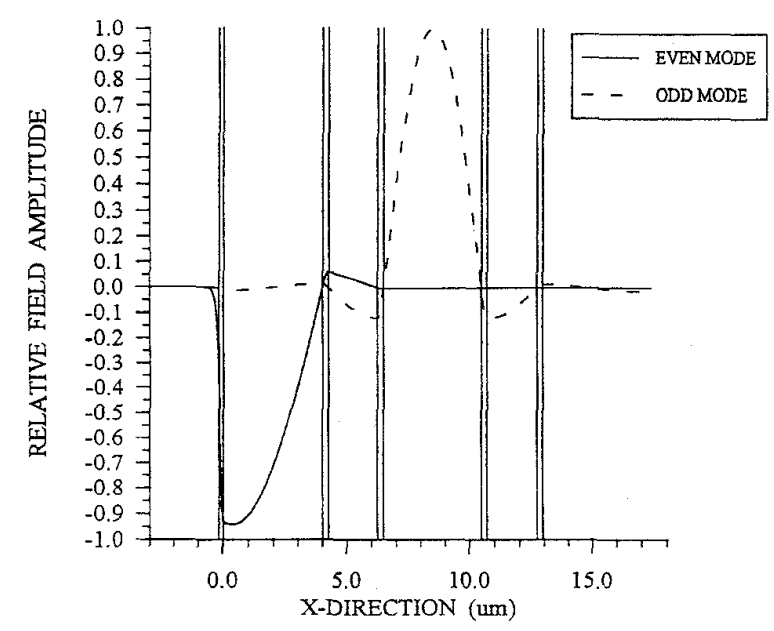

(b)

Fig. 9. E-field for $N_{e}$ and $N_{o}$ with InP substrate: (a) Coupling region as in Fig. 1(b): $d_{l 1}=d_{l 2}=2 \mu \mathrm{m}, d_{g 1}=d_{g 2}=4 \mu \mathrm{m}$, $d_{h_{1}}^{\prime}=d_{h 1}=d_{h, 2}=0.237 \mu \mathrm{m}, n_{l 1}=n_{l 2}=n_{g 1}=n_{g 2}=3.16$, $n_{h 1}=n_{h 2}=3.55$, and $\lambda=1.55 \mu \mathrm{m}$, (b) Decoupling region as in Fig. 1(b): $d_{l 1}=0 \mu \mathrm{m}, d_{h 1}^{\prime}=0.162 \mu \mathrm{m}, d_{l 2}=2 \mu \mathrm{m}, d_{g 1}=d_{g 2}=4 \mu \mathrm{m}$, $d_{h 1}=d_{h 2}=0.237 \mu \mathrm{m}, n_{l 1}=n_{l 2}=n_{g 1}=n_{g 2}=3.16$, $n_{h 1}=n_{h 2}=3.55$, and $\lambda=1.55 \mu \mathrm{m}$.

coupling-length and a decoupling region with $d_{l 1}=0 \mu \mathrm{m}$ and $d_{h 1}^{\prime}=0.162 \mu \mathrm{m}$. BPM simulation results in Fig. 10(b) again show the expected results. Propagating after a coupling-length of $7.34 \mathrm{~mm}$ in the coupling region, light is completely coupled from the upper guide to the lower guide. In the decoupling region optical guide power remain in the lower guide for a long distance $\left(>5 L_{c}=37.5 \mathrm{~mm}\right)$. Since we can easily control coupling-efficiency between dual ARROW waveguides by simply varying the thicknesses of upper cladding layers, the theory and idea can be applied to designing a power divider with any power ratio based on our theory and this will be discussed in detail in another paper.

\section{CONClusion}

We have developed the necessary theory to analyze the coupling efficiency of dual ARROW waveguides. Based on the 

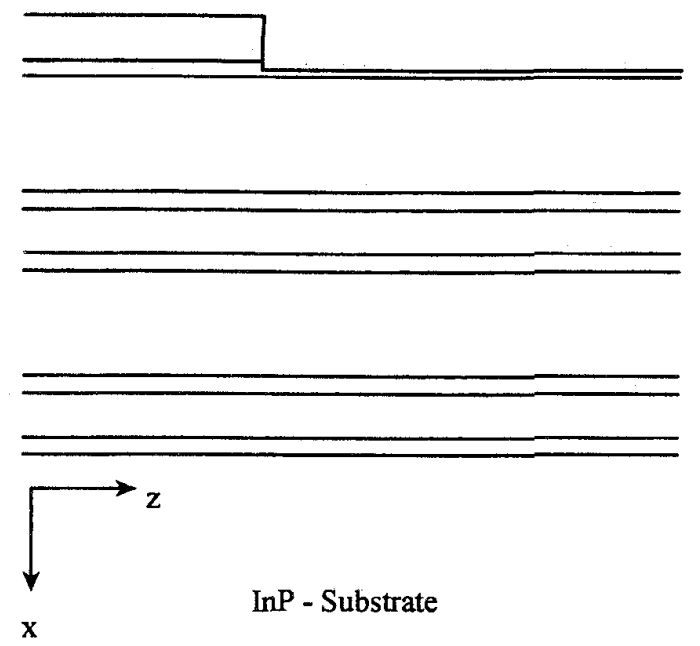

(a)

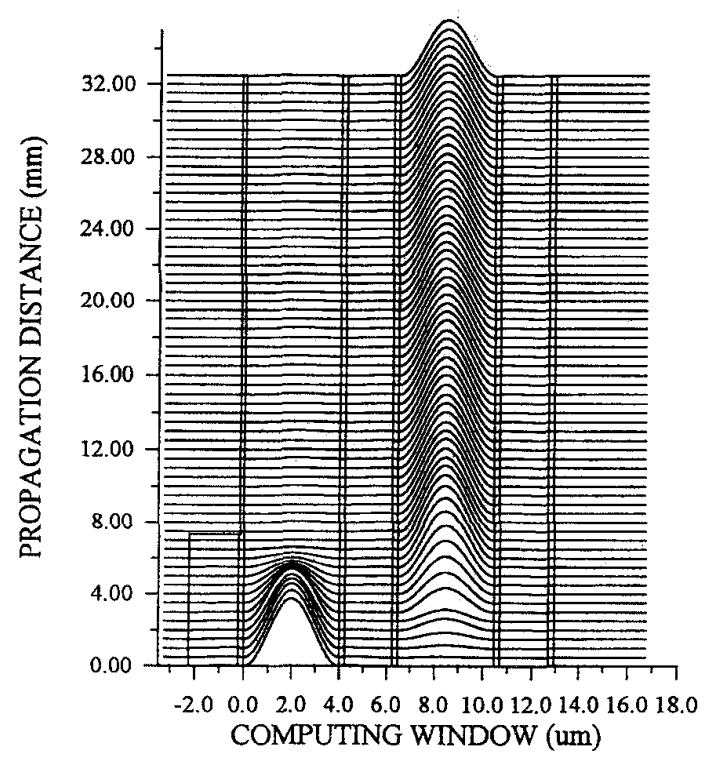

(b)

Fig. 10. (a) The configuration of dual-ARROW switching structure consisting of a coupling and a decoupling region with corresponding parameters in Fig. 9(a) and (b). (b) BPM simulation results with the structure in Fig. 10(a).

theory, we have analyzed the coupling efficiencies of dual ARROW waveguides with different upper cladding thicknesses on $\mathrm{Si}$ and InP substrates. With the same thicknesses of both upper and lower cladding layers to maintain the symmetry feature, the coupling efficiency can be reached to about $100 \%$ with a negligible loss. On the other hand, when the upper cladding layers are almost suitably removed to destroy the symmetry property, the coupling efficiency drops to near zero such that optical power can be confined in the original waveguide also with a negligible loss. BPM simulations also give same results and verify the theory. The theory and simulation results can be efficiently applied to designing an ARROW power divider with any specified power ratio.

\section{ACKNOWLEDGMENT}

The authors would like to thank the reviewers for their helpful suggestions.

\section{REFERENICES}

[1] H. F. Taylor and A. Yariv, "Guided wave optics," in Proc. IEEE, vol. 62, Aug. 1974, pp. $1044-1060$.

[2] E. A. J. Marcatili, "Dielectric rectangular waveguide and directional coupler for integrated optics," Bell Syst. Tech. J., vol. 48, pp. 2071-2102, 1969.

[3] D. Marcuse, "The coupling of degenerate modes in two parallel dielectric waveguides," Bell Syst. Tech. J., vol. 50, pp. 1791-1816, 1971.

[4] H. Kogelnik and R. V. Schmidt, "Switched directional couplers with alternating $\triangle \beta$," IEEE J. Quantum Electron., vol. QE-12, pp. 396-401, July 1976.

[5] D. Marcuse, Light Transmission Optics. New York: Van Nostrand, 1972.

[6] A. Yariv, "Coupled mode theory for guided-wave optics," IEEE J. Quantum Electron., vol. QE-9, pp. 919-933, Sept. 1973.

[7] A. Hardy and W. Streifer, "Coupled mode theory of parallel waveguides," IEEE J. Lightwave Technol, vol. LT-3, pp. 1135-1146, Oct. 1985.

[8] M. A. Duguay, Y. Kokubun, and T. L. Koch, "Antiresonant reflecting optical waveguides in $\mathrm{SiO}_{2}$-Si multilayer structures," Appl. Phys. Lett., vol. 49, pp. 13-15, Jan. 1986.

[9] T. Baba, Y. Kokubun, T. Sakaki, and K. Iga, "Loss reduction of an ARROW waveguide in shorter wavelength and its stack configuration," IEEE J. Lightwave Technol., vol. 6, pp. 1440-1444, Sept. 1988.

10] M. Mann, U. Trutschel, C. Wachter, L. Leine, and F. Lederer, "Directional coupler based on an antiresonant reflecting optical waveguide," Opt: Lett., vol. 16, no. 11, pp. 805-307, June 1991.

[11] J. M. Kubica, "Numerical analysis of InP/InGaAsP ARROW waveguides using transfer matrix approach," IEEE J. Lightwave Technol., vol. 10, pp. 707-771, June 1992.

[12] Y. Suematsu and K. Kishino, "Coupling coefficient in strongly coupled dielectric waveguides," Radio Science, vol. 12, no. 4, pp. 587-592, July-Aug. 1977.

[13] B. Broberg and S. Lindgren, "Refractive index of $\mathrm{In}_{1-x} \mathrm{Ga}_{x} \mathrm{As}_{y} \mathrm{P}_{1-y}$ layers and InP in the transparent wavelength region," J. Appl. Phys., vol. 55, no. 9, pp. 3376-3381, May 1984.

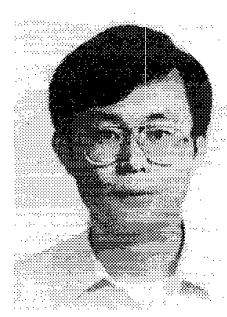

Yeong-Her Chen ('S'95) received the B.S. degree from Chung Yuan University in 1989, and the M.S. degree from National Tsing Hua University in 1991, both in elestrical engineering. $\mathrm{He}$ is currently working toward the Ph.D. degree in electronics at National Chiao Tung University. His current reseatch is focused on integrated optical elements for optical interconnect and optical communication applications.

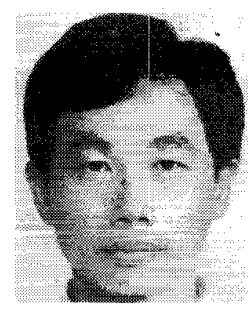

Yang-Tung Huang (M'82) received the B.S. degree in electrophysics and the M.S. degree in electronics form National Chiao Tung University in 1978 and 1982, respectively, and the Ph.D. degree in electrical and computer engineering from the University of Arizona in 1990 .

He joined the faculty at National Chiao Tung University in 1983, where he currently holds the position of Professior at the Department of Electronics Engineering and the Institute of Electronics. He is also Deputy Director of the Semiconductor Research Center. His current research interests include integrated optics, holography, and optical interconnects. 